\title{
Remarks on A. B. Turowicz's and Z. Mikołajska's notes on approximations to roots of positive numbers
}

\author{
by P. H. Diananda (Singapore)
}

1. In a recent note [3] Turowiez proved the following result (called $T$, hereafter):

Let $A$ and $x_{0}$ be positive and $n$ be an integer $>1$. Then the sequence $\left\{x_{r}\right\}(r \geqslant 0)$, where

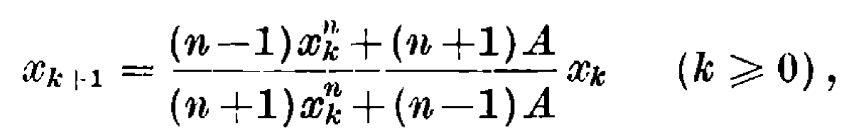

converges to $A^{1 / n}$.

In another recent note [1] Mikołajska, using Turowicz's method, generalized his result to the following (called $\boldsymbol{M}$, hereafter):

Let $A$ and $x_{0}$ be positive. Then the sequence $\left\{x_{r}\right\}$, where

$$
x_{k+1}=\frac{\psi\left(x_{k}\right)-\varphi\left(x_{k}^{n}-A\right)}{\psi\left(x_{k}\right)+\varphi\left(x_{k}^{n}+A\right)} x_{k} \quad(k \geqslant 0)
$$

converges to $A^{1 / n}$ if, for non-negative $x$ and real $u, \psi(x)$ and $\varphi(u)$ are continuous,

$$
\begin{aligned}
\psi(x)+\varphi\left(x^{n}-A\right) & >0, \\
\psi(x)-\frac{x+A^{1 / n}}{x-A^{1 / n}} \varphi\left(x^{n}-A\right) & >0 \quad\left(x^{n} \neq A\right),
\end{aligned}
$$

(5) $\quad \varphi(u)=-\varphi(-u), \quad p(u)$ is monotonic and $\operatorname{sgn} \varphi(u)=\operatorname{sgn} u$.

The condition $\operatorname{sgn} \varphi(u)=\operatorname{sgn} u$ is not explicitly mentioned by Mikolajska but it is used in the derivation of the set of equations (9) of her note [1].

In this note we shall prove the following result (called $\boldsymbol{O}$, hereafter):

Let $a$ and $x_{0}$ be positive. Then the sequence $\left\{x_{r}\right\}$, where

$$
x_{k+1}=\frac{\psi\left(x_{k}\right)-\chi\left(x_{k}\right)}{\psi\left(x_{k}\right)+\chi\left(x_{k}\right)} x_{k} \quad(k \geqslant 0)
$$


converges to a, the unique positive zero of $\chi(x)$, if, for positive $x$,

$$
\begin{gathered}
\psi(x) \text { and } \chi(x) \text { are continuous, } \\
\psi(x)>\chi(x)
\end{gathered}
$$

and

$$
\psi(x)>\frac{a}{x-a} \chi(x)>0 \quad(x \neq a) .
$$

It is not difficult to prove that $\boldsymbol{O}$ is a generalization of $\boldsymbol{M}$ and thus of $\boldsymbol{T}$ too. For this purpose, set

$$
a=A^{1 / n} \quad \text { and } \quad \chi(x)=\varphi\left(x^{n}-A\right)
$$

and let the conditions in $M$ be satisfied. Then we clearly have (6) from (2), and also (7) from the continuity of $\psi(x)$ and $\varphi(u)$.

From (3) to (5) and (10), we have, for positive $x$,

$$
\begin{gathered}
\psi(x)+\chi(x)>0, \\
\psi(x)-\frac{x+a}{x-a} \chi(x)>0 \quad(x \neq a)
\end{gathered}
$$

and

$$
\operatorname{sgn} \chi(x)=\operatorname{sgn}(x-a) .
$$

Adding (11) and (12) and using (13), we clearly have (9). We have $\psi(x)>-\chi(x)$, from (11); and $\chi(x) \leqslant 0$ for $x \leqslant a$, from (13). Thus

$$
\psi(x)>-\chi(x) \geqslant 0 \geqslant \chi(x) \quad(x \leqslant a) .
$$

Hence (8) is true for $x \leqslant a$. For $x>a,(8)$ follows from (12) and (13).

Thus (6) to (9) are all satisfied. Hence $\boldsymbol{O}$ generalizes $\boldsymbol{M}$ and $\boldsymbol{T}$.

2. To prove $O$, we first prove (11) and (13). From (7) and (9), we have (13) and, in particular,

$$
\chi(a)=0 .
$$

For $x=a,(11)$ follows from (8) and (14). Also

$$
\psi(x)+\chi(x)>\frac{x}{x-a} \chi(x)>0 \quad(x \neq a),
$$

from (9). Thus (11) is true. Note that (13) implies that $a$ is the unique positive zero of $\chi(x)$.

We next prove that if $x_{s}=a$ for some $s \geqslant 0$ then $x_{r}=a$ for all $r \geqslant 8$. This follows, by induction on $r$, since $x_{k}=a$ implies $x_{k+1}=a$ from (6), (11) and (14). Hence $O$ follows if some $x_{s}=a$, the unique positive zero of $\chi(x)$. 
In what follows we therefore assume that no $x_{s}=a$. From (6),

$$
\frac{x_{k+1}-a}{x_{k}-a}=\frac{\psi\left(x_{k}\right)-\frac{x_{k}+a}{x_{k}-a} \chi\left(x_{k}\right)}{\psi\left(x_{k}\right)+\chi\left(x_{k}\right)} \quad(k \geqslant 0)
$$

as may be verified. Thus

if, for positive $x$,

$$
\left|x_{k+1}-a\right|<\left|x_{k}-a\right| \quad(k \geqslant 0)
$$

$$
-\psi(x)-\chi(x)<\psi(x)-\frac{x+a}{x-a} \chi(x)<\psi(x)+\chi(x) \quad(x \neq a) .
$$

But (17) follows from (9), since (17) is equivalent to

$$
\psi(x)>\frac{a}{x-a} \chi(x) \quad \text { and } \quad \frac{x}{x-a} \chi(x)>0 \quad(x \neq a) .
$$

Hence (16) is true. Thus $\left\{\left|x_{r}-a\right|\right\}$ is a positive decreasing sequence. Therefore it converges to a non-negative limit $L$. $O$ follows if $L$ is zero, since $a$ is the unique positive zero of $\chi(x)$.

Suppose that $L$ is non-zero. Then $L$ is positive and the sequence $\left\{x_{r}\right\}$ has a subsequence $\left\{x_{u_{r}}\right\}\left(0 \leqslant u_{0}<u_{1}<\ldots\right)$, which converges to a finite limit $c=a \pm L \neq a$. Also $c$ is positive since the $x_{r}$ and $a$ are all positive and $\left\{\left|x_{r}-a\right|\right\}$ is a decreasing sequence.

The sequence $\left\{x_{u_{r}}\right\}$ converges to $c$, positive and not $a$. Hence using (7), (11), (15) and considerations of continuity, we obtain that the sequence $\left\{x_{u_{r} ; 1}\right\}$ converges to a limit $d$ satisfying

$$
\frac{d-a}{c-a}=\frac{\psi(c)-\frac{c+a}{c-a} \chi(c)}{\psi(c)+\chi(c)}
$$

Since $c$ is positive and not $a$, it follows, in the same way as (16), that

$$
|d-a|<|c-a| \text {. }
$$

But the sequence $\left\{\left|x_{r}-a\right|\right\}$ converges to $L$ and therefore its subsequences $\left\{\left|x_{u_{r}}-a\right|\right\}$ and $\left\{\left|x_{u_{r}+1}-a\right|\right\}$ also converge to $L$. Hence $|c-a|=L$ and $|d-a|=L$. This contradicts (18). Thus our supposition that $L$ is nonzero is false. Hence $L$ is zero.

3. Turowicz and Mikolajska proved that the sequences $\left\{x_{r}\right\}$, in $\boldsymbol{T}$ and $M$, not merely converge, but converge monotonically, to $A^{1 / n}$. The conditions assumed in $\boldsymbol{O}$ do not ensure that the sequence $\left\{x_{r}\right\}$, in $O$, is monotonic. However, this can be ensured if we replace the conditions (8) and (9) by

$$
\psi(a)>0 \quad \text { and } \quad \psi(x)>\frac{x+a}{x-a} \chi(x)>0 \quad(x \neq a) .
$$


To prove this, suppose that (8) and (9) in $O$ have been replaced by (19). Clearly (19) implies (9); (7) and (19) imply (13); and (13) and (19) imply (8). Thus the conditions of $\boldsymbol{O}$ are all satisfied. Consequently, the sequence $\left\{x_{r}\right\}$ converges to $a$. Also, as in $\S 2,(11)$ and (15) are true. From (11), (15) and (19) we obtain that all the $x_{r}-a$ have the same sign. Thus, since the sequence $\left\{x_{r}\right\}$ converges to $a$, the convergence is monotonic.

4. In $M, n$ is not restricted to be an integer while, in $T$, there is this restriction. We can remove it by calculus methods, noting that identification of (1), (2) and (6) yields

$$
\psi(x)=n\left(x^{n}+A\right) \quad \text { and } \quad \varphi\left(x^{n}-A\right)=\chi(x)=x^{n} \cdots A,
$$

where $A=a^{n}$, and using $M$ or our result of $\S 3$.

Turowicz also proved that the sequence $\left\{x_{r}\right\}$, defined by

(20) $x_{k+1}=\frac{(n-1)(2 n-1) x_{k}^{2 n}+2\left(4 n^{2}-1\right) A x_{k}^{n}+(n+1)(2 n+1) A^{2}}{(n \div 1)(2 n+1) x_{k}^{2 n}+2\left(4 n^{2}-1\right) A x_{k}^{n}+(n-1)(2 n-1) A^{2}} x_{k}(k \geqslant 0)$,

where $x_{0}$ and $A$ are positive and $n$ is an integer $>1$, converges to $A^{1 / n}$. For this sequence, identification of (2), (6) and (20) yields

and

$$
\psi(x)=\left(2 n^{2}+1\right)\left(x^{2 n}+A^{2}\right)+2\left(4 n^{2}-1\right) A x^{\prime \prime}
$$

$$
\varphi\left(x^{n}-A\right)=\%(x)=3 n\left(x^{2 n}-A^{2}\right),
$$

where $A=a^{n}$. It is seen that $\varphi(u)=3 n\left(u^{2}+2 A u\right) \neq-\varphi(-u)$. Thus (5) is not satisfied and $M$ is inapplicable. However, we can apply our result of $\S 3$ to prove that the sequence converges monotonically to $A^{1 / n}$ for real (not merely integral) $n>1$.

The extensions of Turowicz's results for non-integral $n$ are not of practical importance since $x_{k}^{n}$ has to be first computed to find $x_{k+1}$.

5. Turowicz and Mikołajska proved results on the rapidity of convergence of the sequences $\left\{x_{r}\right\}$ in $\boldsymbol{T}$ and $\boldsymbol{M}$. Following them, using (15), we can easily prove, for the convergent sequence $\left\{x_{r}\right\}$ in $O$, the following:

Let $\inf _{E}|\psi(x)+\chi(x)|=\sigma>0$, where $E=\left\{x: x>0,|x-a| \leqslant\left|x_{0}-a\right|\right\}$, and $\sup _{E}|(x-a) \psi(x)+(x+a) \chi(x)||x-a|^{-\nu}=\tau(\nu)<\infty \quad(\nu$ real $)$. Then

$$
\left|x_{k+1}-a\right| \leqslant \frac{\tau(\nu)}{\sigma}\left|x_{k}-a\right|^{\nu} \quad(k \geqslant 0)
$$

and the convergence of $\left\{x_{r}\right\}$ is of degree $\geqslant v$. Further, this convergence is of degree $\geqslant N$ if $N$ is the largest $v$ for which $\tau(v)<\infty$. 
For the monotonically convergent sequence $\left\{x_{r}\right\}$ in our result of $\S 3$, omitting the trivial case $x_{0}=a$, we can improve the above by replacing the set $E$ by the set $F=\left\{x: 0<\frac{x-a}{x_{0}-a} \leqslant 1\right\}$.

6. Richmond [2] had, previously to Turowicz, studied the sequence in $T$, given references to earlier work and stated that the case $n=3$ was given by $\mathbf{R}$. Burrow in his Theory of Gunnery published in $\mathbf{1 7 7 9}$. Richmond also studied the sequence $\left\{x_{r}\right\}$, where $x_{0}$ is suitably chosen and

$$
x_{k+1}=x_{k}-\frac{2 \chi\left(x_{k}\right) \chi^{\prime}\left(x_{k}\right)}{2\left\{\chi^{\prime}\left(x_{k}\right)\right\}^{2}-\chi\left(x_{k}\right) \chi^{\prime \prime}\left(x_{k}\right)} \quad(k \geqslant 0) .
$$

This sequence results from the application of Newton's method of approximation to find a zero of the function $f(x)=\chi(x)\left\{\chi^{\prime}(x)\right\}^{-1 / 2}$. Richmond has shown that, under certain conditions, the convergence of this sequence to a zero of $\chi(x)$ is of degree 3. Further, if $x_{0}$ is positive, this sequence (i) results from that in $O$ if

$$
\psi(x)=\frac{x^{-1} \chi^{\prime}(x)}{2\left\{\chi^{\prime}(x)\right\}^{2}-\chi(x) \chi^{\prime \prime}(x)}-\chi(x)
$$

and (ii) as noted by Richmond, reduces to that in $T$ if $\chi(x)=x^{n}-A$.

\section{References}

[1] Z. Mikołajska, Remarque sur la note de A. B. Turowicz sur l'approximation des racines de nombres positifs, Ann. Polon. Math. 8 (1960), pp. 285-289.

[2] H. W. Richmond, On certain formulae for numerical approximation, J. London Math. Soc. 19 (1944), pp. $31-38$.

[3] A. B. Turowicz, Sur l'approximation des racines de nombres positifs, Ann. Polon. Math. 8 (1960), pp. 265-269.

DEPARTMENT OF MATHEMATICS, UNIVERSITY OF SINGAPORE

Reģu par la Rédaction le 2.5.1963 